\title{
Fetal Anatomical Survey during Second Trimester Screening Examination
}

\author{
Vincenzo D'Addario, Vincenzo Pinto, Luca Di Cagno, Armando Pintucci, Angela Cristina Rossi
}

\begin{abstract}
Screening for fetal malformations is one of the main aims of ultrasonography during pregnancy. In most cases fetal anomalies occur as an unexpected event in not at risk patients: For this reason the only way to rule out congenital malformations is to screen every pregnant patient with a systematic evaluation of the fetal anatomy. The ultrasonic screening of fetal malformations has to be based on a single examination that has to be planned at a gestational age which is advanced enough to visualize most fetal anomalies and early enough to plan further diagnostic tests and allow the termination of pregnancy in the case of severe malformation. The gestational age that constitutes the best compromise between the need of an early diagnosis and the natural history of most congenital malformations is the period ranging from 19 to 21 weeks of gestation. This ultrasonic examination is known as 'fetal anomaly scan' or 'fetal morphology scan'. Some scientific societies suggest guidelines for the optimal performance of such examination. In this paper the minimal standards for an accurate 'fetal morphology scan' will be described.
\end{abstract}

Keywords: Fetus, Ultrasound, Fetal anatomy, Fetal malformations, Screening.

How to cite this article: D'Addario V, Pinto V, Di Cagno L, Pintucci A, Rossi AC. Fetal Anatomical Survey during Second Trimester Screening Examination. Donald School J Ultrasound Obstet Gynecol 2012;6(1):43-54.

Source of support: Nil

Conflict of interest: None declared

\section{INTRODUCTION}

Screening for fetal malformations is one of the main aims of ultrasonography during pregnancy. In most cases, fetal anomalies occur as an unexpected event in not at risk patients: for this reason the only way to rule out congenital malformations is to screen every pregnant patient with a systematic evaluation of the fetal anatomy.

Fetal organogenesis is a complex and evolutive process that cannot be squeezed in a single ultrasonic examination; ${ }^{1}$ an accurate evaluation, which would take in account the complex fetal morphological evolution, should require multiple ultrasonic examinations during pregnancy. In the clinical practice, however, a so complicated screening program cannot be applied to the general population of the pregnant women due to the elevated cost and the unjustified use of medical resources. For this reason, the ultrasonic screening of fetal malformations has to be based on a single examination that has to be planned at a gestational age which is advanced enough to visualize most fetal anomalies and early enough to plan further diagnostic tests and allow the termination of pregnancy in the case of severe malformation. The gestational age that constitutes the best compromise between the need of an early diagnosis and the natural history of most congenital malformations is the period ranging from 19 to 21 weeks of gestation. This ultrasonic examination is known as 'fetal anomaly scan' or 'fetal morphology scan'.

In order to obtain a systematic evaluation of the fetal anatomy it is essential to establish:

- The orientation of the ultrasonic images on the screen in regard to the patient lie (i.e. the left border of the screen corresponds to the left side of the patient abdomen in the axial scans and to the cephalic pole in the longitudinal ones)

- The fetal lie in the uterus.

To obtain the latter goal it is necessary to correlate the three-dimensional movements of the probe on the maternal abdomen to the bidimensional images appearing on the screen and to reconstruct a mental three-dimensional model from the pool of images seen. Once the idea of the fetal lie in uterus is obtained, the probe can be quickly oriented on the correct plane to visualize the desired fetal anatomical structure. $^{2}$

The fetal anatomical figures, which can be visualized by ultrasound, are innumerable and the expert sonographer may carry out a detailed evaluation of the complex fetal anatomy.

\section{HEAD AND BRAIN}

The measurements of biparietal diameter (BPD) and head circumference are obtained on an axial scan of the fetal head showing the thalami, the cavum septi pellucidi and the frontal horns of the lateral ventricles (transthalamic scan) (Fig. 1).

By slightly tilting the probe caudally (toward the base of the skull), the atrium of the lateral ventricle filled by the echogenic choroid plexus and continuing in the posterior occipital horn is shown (transventricular scan) (Fig. 2); at this level the atrial width is measured: Its normal value is less than $10 \mathrm{~mm}$ independently from the gestational age. During the second trimester, the surface of the cerebral hemispheres is still smooth, since the development of the 


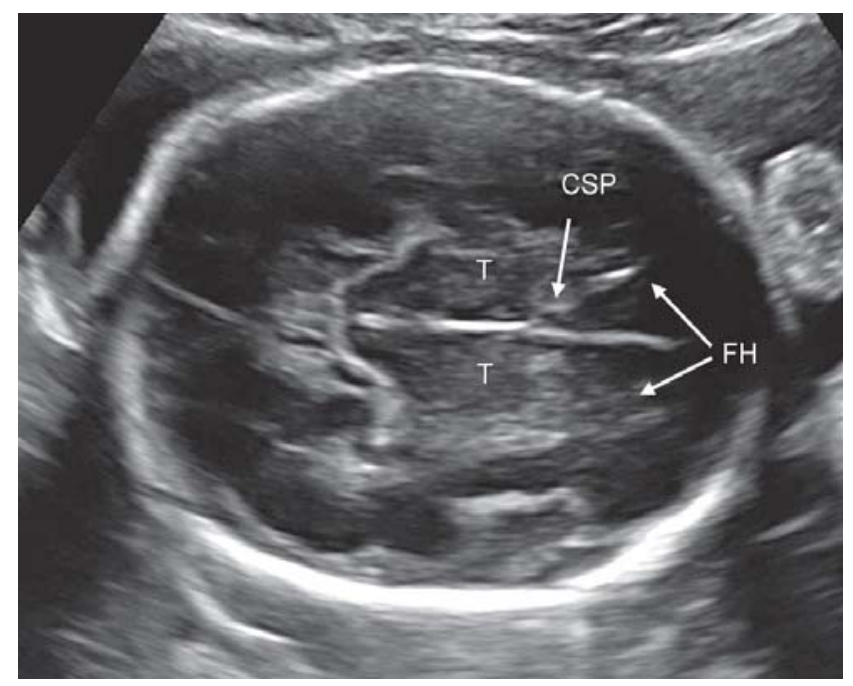

Fig. 1: Transthalamic scan for measurement of BPD and HC. $\mathrm{T}$ : thalami; CSP: cavum septi pellucidi; $\mathrm{FH}$ : frontal horns

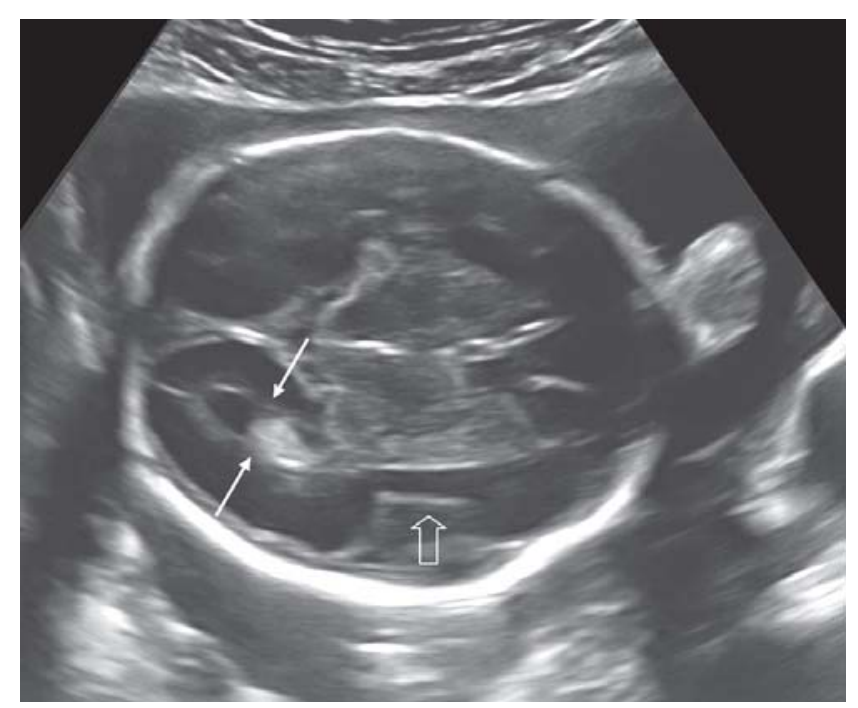

Fig. 2: Transventricular scan for measurement of the atrial width. Arrows point to the atrium of the lateral ventricle. Arrowhead = insula

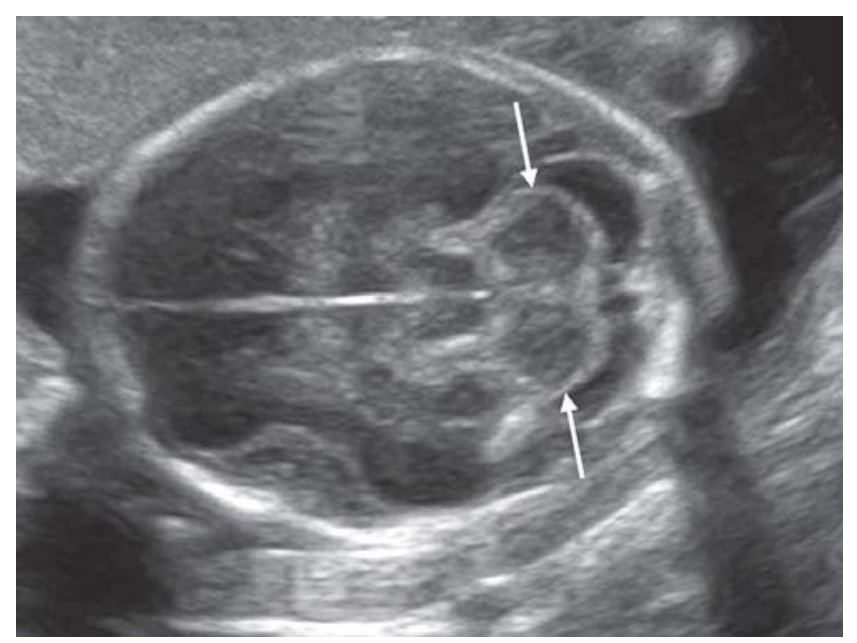

Fig. 3: Transcerebellar scan for measurement of the transverse cerebellar diameter (arrows). The cerebellum is contourned by the cisterna magna

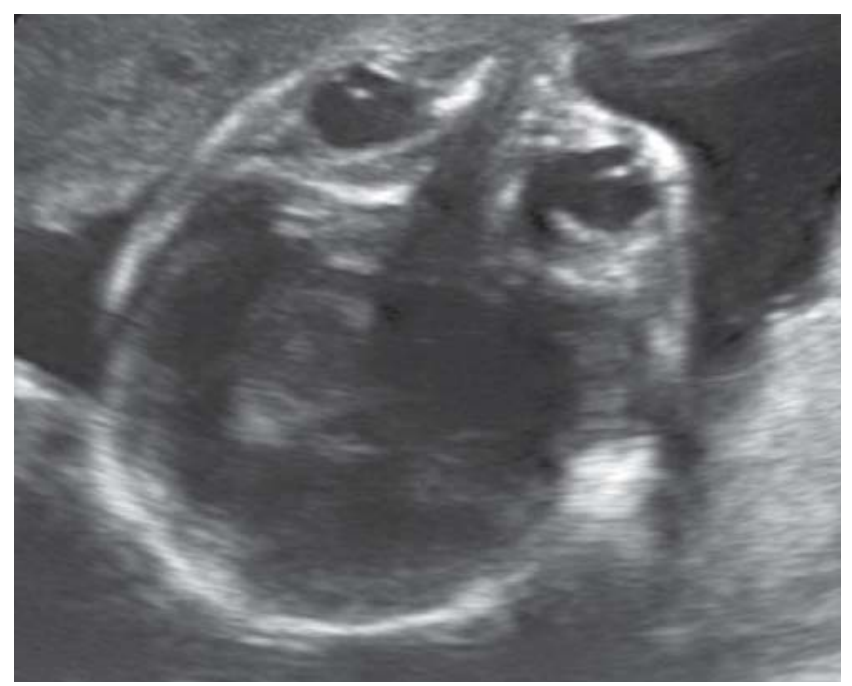

Fig. 4: Transverse scan on the orbits. The lenses can be recognized

gyri and sulci is a late event, and the Sylvian fissure is wide, such allowing to recognize the lobe of the insula.

By further tilting the probe caudally, the posterior fossa is shown with the cerebellum and the cisterna magna (transcerebellar scan) (Fig. 3). At this level the transverse cerebellar diameter is measured. By tilting the probe on the opposite side, the orbits can be visualized (transorbitary scan) (Fig. 4).

These four simple scans allow an accurate evaluation of the brain anatomy and the recognition of the majority of the brain anomalies. However, further scans can be obtained allowing a more detailed evaluation of the finest brain structures. Particularly when the fetus is in breech presentation or in transverse lie, sagittal and coronal scans can be obtained. A midsagittal scan shows the corpus callosum above the cavum septi pellucidi as well as the posterior fossa, where the cerebellar vermis and the fourth ventricle can be recognized (Fig. 5). A parasagittal scan shows the different components of the lateral ventricle (so called 'three horns view') (Fig. 6). A more external parasagittal scan shows the smooth surface of the brain with the wide insula delimited by the opercula (Fig. 7). The coronal scans will show the lateral ventricles with a different appearance, depending on the level of the scan: the most anterior one will show the frontal horns in the frontal hemispheres (transfrontal plane) (Fig. 8A); moving behind the cavum septi pellucidi and corpus callosum may be seen interposed between the frontal horns above the caudate nuclei (transcaudate plane) (Fig. 8B); then the thalami may be seen below the bodies of the lateral ventricles (transthalamic plane) (Fig. 8C) and finally the occipital horns with their typical round appearance above the cerebellum may be seen in the most posterior plane (transcerebellar plane) (Fig. 8D). 


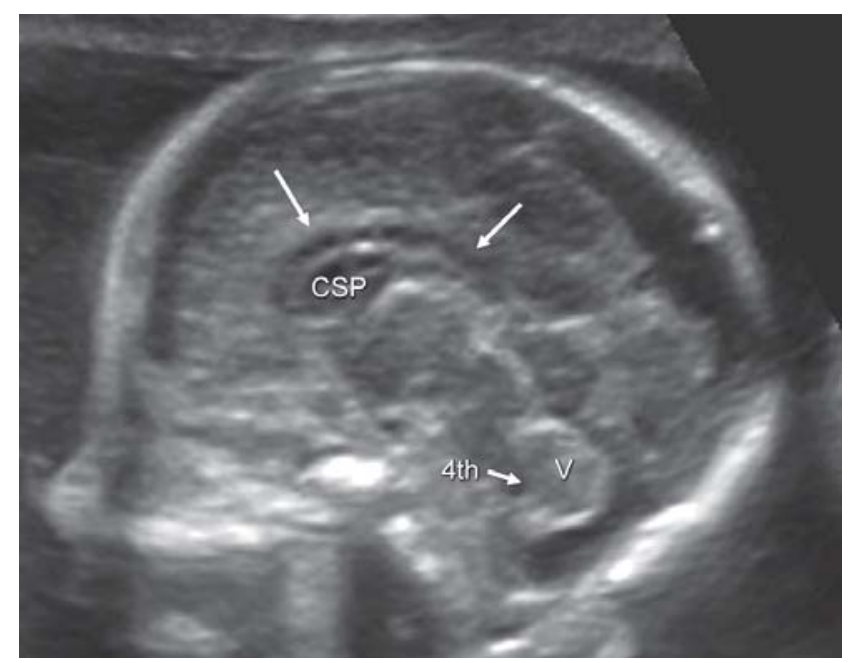

Fig. 5: Midsagittal scan of the brain showing the corpus callosum (arrows), the cavum septi pellucidi (CSP), the posterior fossa with the cerebellar vermis $(\mathrm{V})$ and the fourth ventricle (4th)

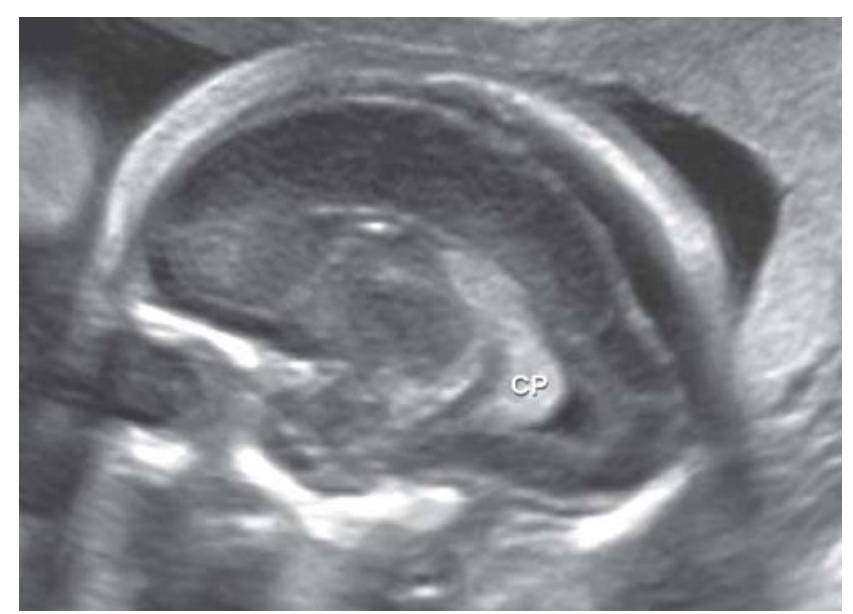

Fig. 6: Parasagittal scan of the brain showing the 'three horns view' of the lateral ventricle with the choroid plexus inside (CP)

The fetal face can be evaluated both by midsagittal scan, showing the profile (Fig. 9) and by coronal scan, showing the lips and nostrils (Fig. 10).

Axial scans at the level of the mouth show the maxilla in the upper level and the tongue with the pharynx at the lower level (Figs 11A and B).
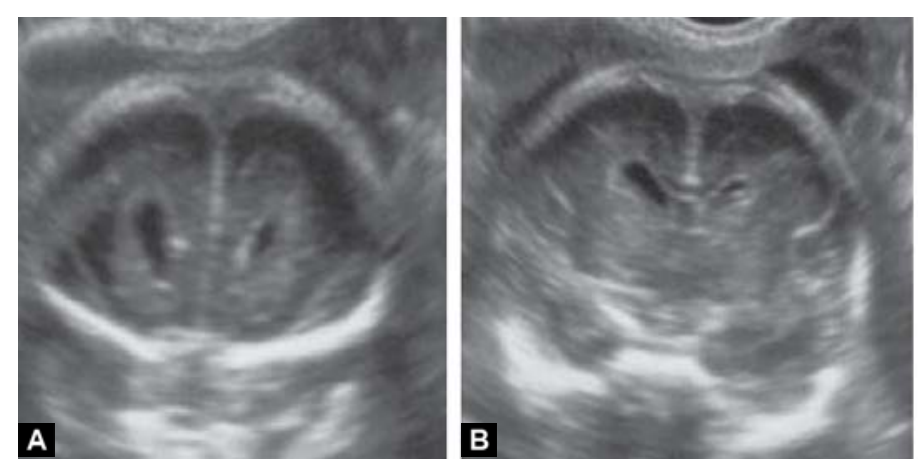

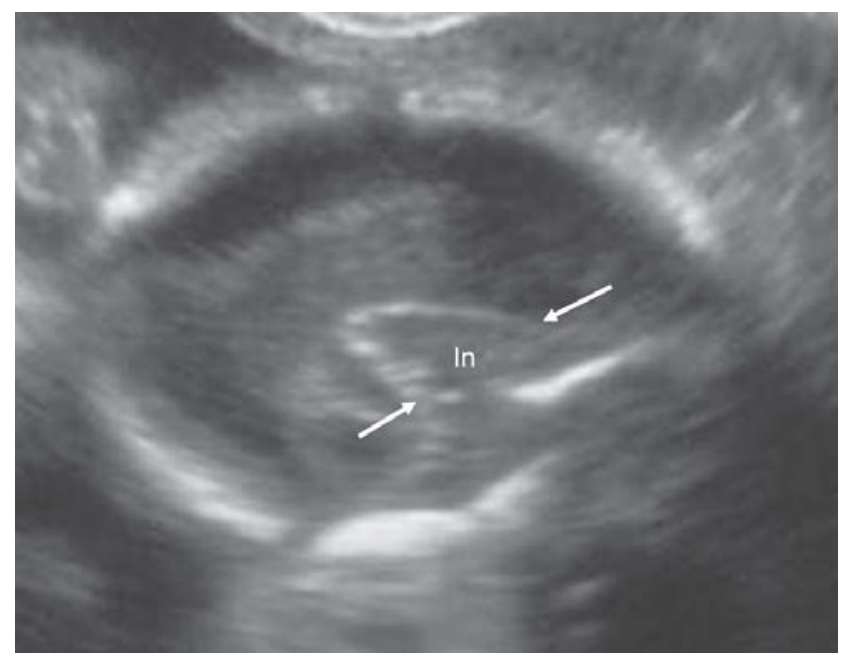

Fig. 7: External parasagittal scan showing the smooth surface of the brain, the wide insula (In) and the opercula (arrows)

\section{SPINE}

The spine can be evaluated in longitudinal, coronal and axial scans. The longitudinal scan passing through the spinal canal may also show the chest and ribs which produce sharp acoustic shadows (Fig. 12). An alternative way to visualize the spinal canal is the coronal scan passing through the laminae (Fig. 13).

In the axial scan, the vertebrae show three ossification centers independently from the level, one generating the body and two generating the laminae, which will fuse posteriorly in the spinous process. According to the level of the section, the axial view may show the clavicle, the ribs or the kidneys (Figs 14A to C).

Close to the spine, further bony structures can be seen, such as the scapulae and the iliac wings (Figs 15A and B).

\section{CHEST}

It is important to establish the correct fetal position in utero in order to confirm the normal 'situs solitus' (both stomach and apex of the heart on the left side of the fetus).

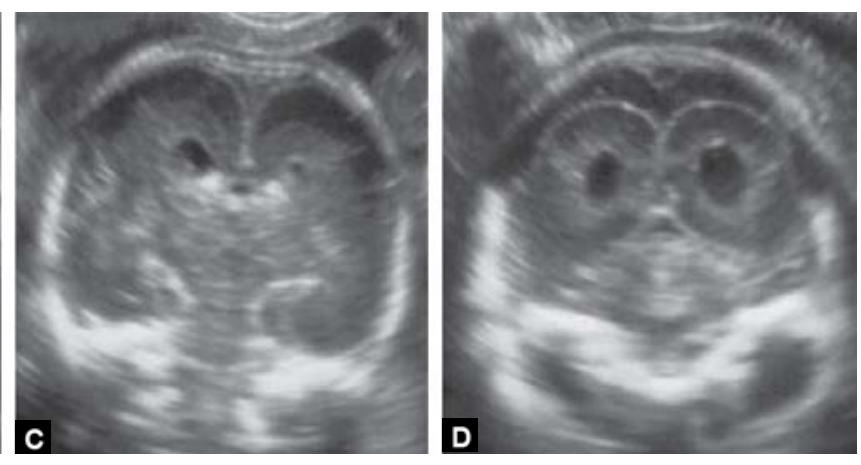

Figs 8A to D: Coronal planes of the brain from the anterior to the posterior aspect: (A) Transfrontal;

(B) Transcaudate; (C) Transthalamic; (D) Transcerebellar 


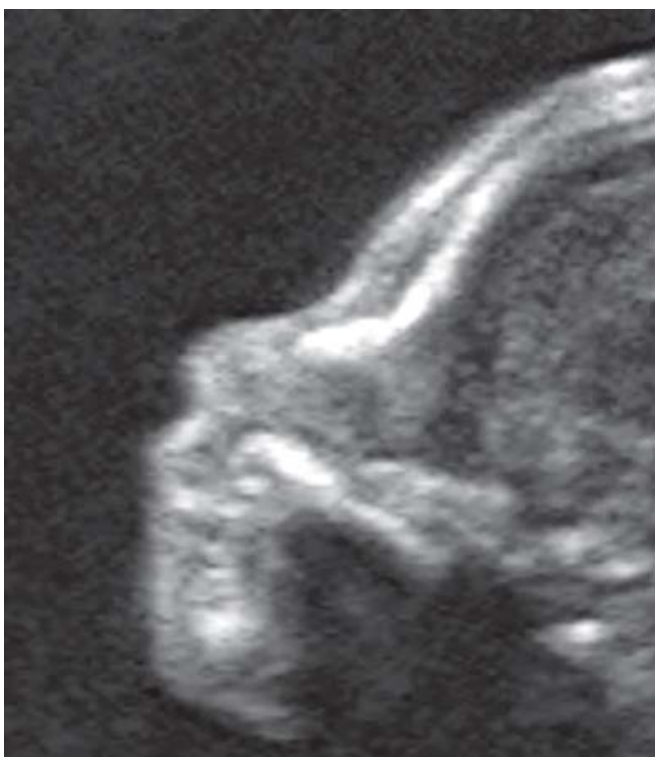

Fig. 9: Fetal profile

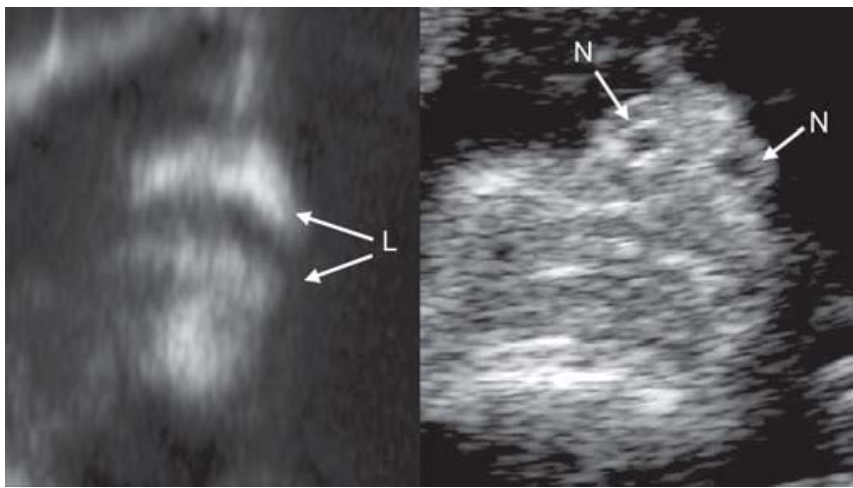

Fig. 10: Coronal scan of the lips $(\mathrm{L})$ and nostrils $(\mathrm{N})$

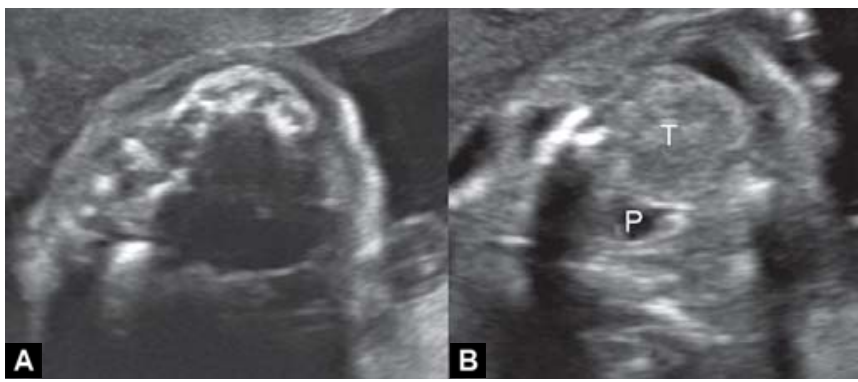

Figs 11A and B: Axial scan of the mouth: (A) At the upper level, the maxilla can be seen; (B) At the lower level, the tongue $(T)$ and the pharynx $(P)$ are shown

The best section to evaluate the chest structures is the axial one at the level of the heart: This scan shows the hyperechoic lungs 'embracing' the cardiac area. In this axial scan, the four-chambers view of the heart is looked for. In order to obtain a correct four-chambers view, the first step is to localize the stomach in an axial scan on the abdomen and then moving the transducer cranially without tilting it; in such a way, a confirmation of a normally left-sided heart as well as a correct heart section are easily obtained. The four-chamber view can be 'apical' when the ultrasonic beam

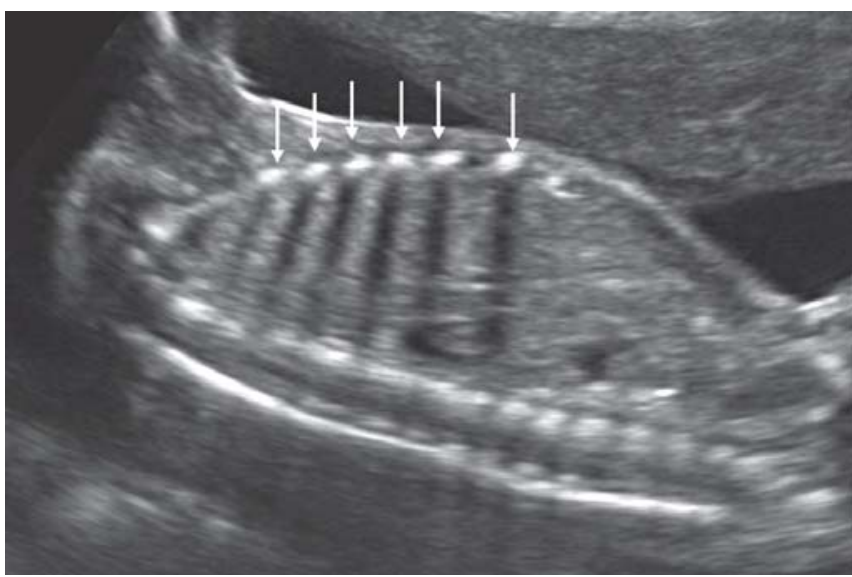

Fig. 12: Longitudinal scan of the spine. The ribs (arrows) produce sharp acoustic shadows

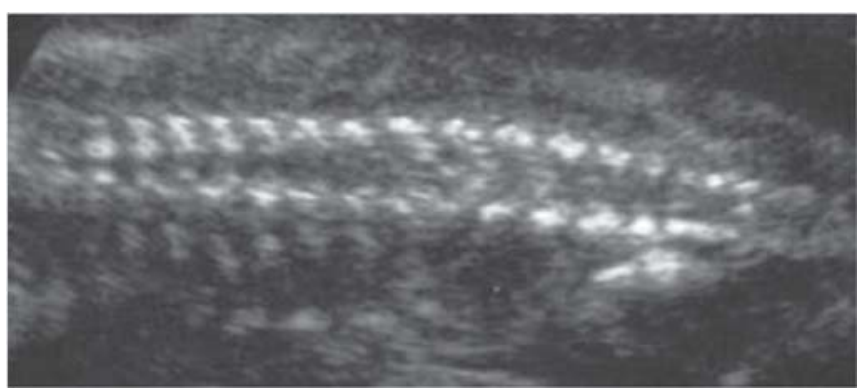

Fig. 13: Coronal scan of the spine passing through the laminae and showing the spinal canal
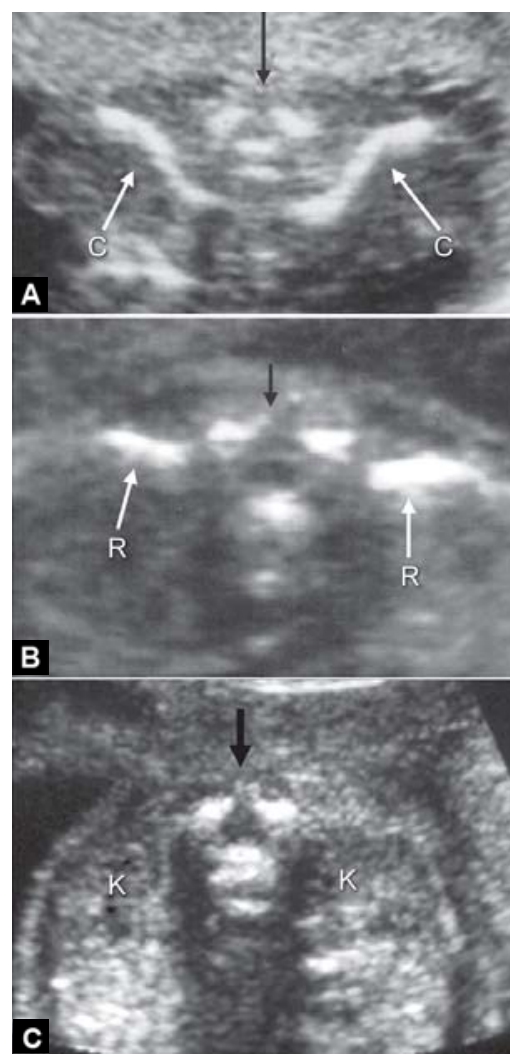

Figs 14A to $C$ : Transverse scan of the vertebrae at different levels: (A) Cervical level, where the clavicles (C) can be seen; (B) Dorsal level, where the ribs $(R)$ can be seen; $(C)$ Lumbar level where the kidneys $(K)$ can be seen. Independently from the level, the vertebrae (black arrows) show three ossification centers referring to the body and the laminae 


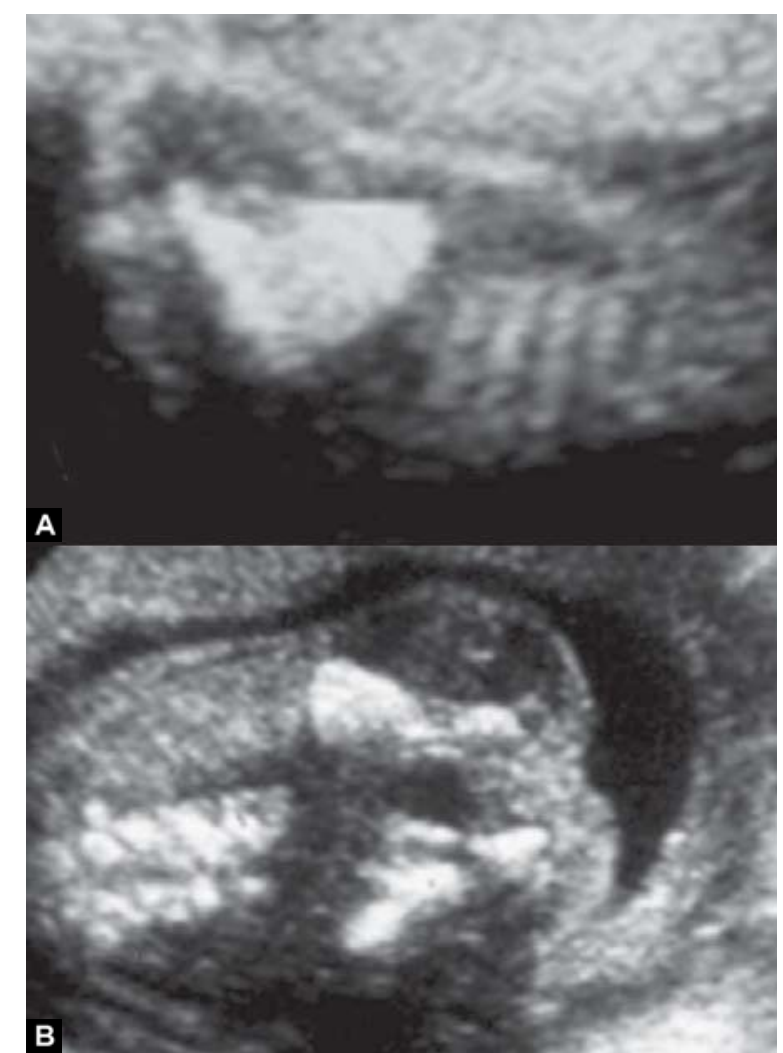

Figs 15A and B: The scapula and the iliac wings can be seen close to the spinal canal

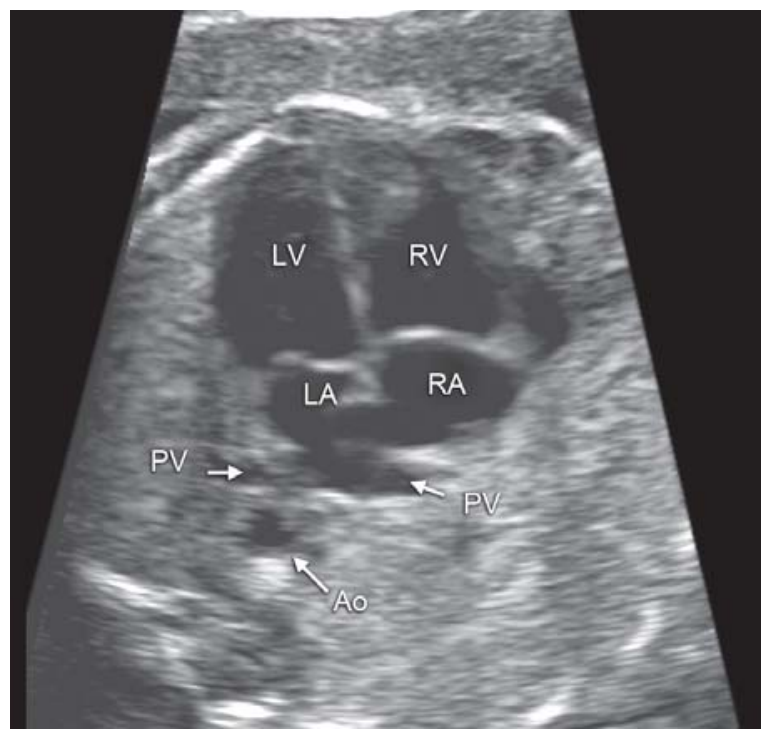

Fig. 16: Four-chambers apical view of the heart: LV: left ventricle; $\mathrm{RV}$ : right ventricle; LA: left atrium; RA: right atrium; PV: pulmonary veins; Ao: descending aorta

is parallel and 'transverse' when it is perpendicular to the interventricular septum. It is better to try to obtain both scans, since the 'apical' is optimal for evaluating the atrioventricular valves and the 'transverse' for evaluating the interventricular and interatrial septa.

In the 'four-chambers' view, the following cardiac structures can be seen (Fig. 16):

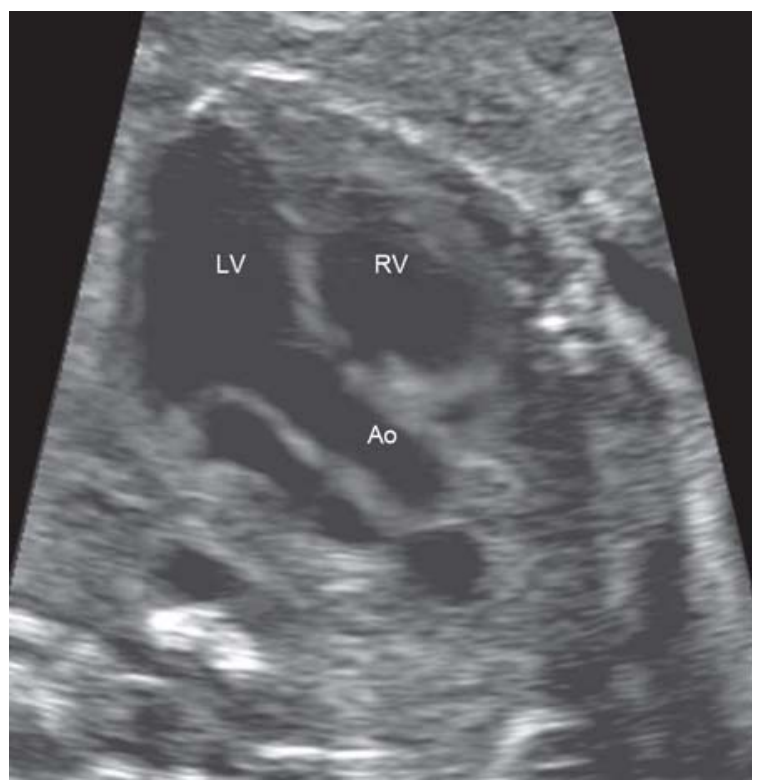

Fig. 17: Left long axis of the heart showing the emerging aorta. $\mathrm{RV}$ : right ventricle; LV: left ventricle; Ao: aorta

- The atria: They have approximately the same size; the left one is closer to the spine and contains the foramen ovale's valve

- Two of the pulmonary veins opening in the left atrium

- The interatrial septum with the interruption due to the foramen ovale

- The atrioventricular valves: The tricuspid has a slightly lower septal insertion than the mitral valve

- The ventricles: They have approximately the same size but a different shape; the right one is more roundish and contains the 'moderator band' close to the apex

- The interventricular septum.

Once the 'four-chambers' view has been obtained, by slight movements of the transducer, it is possible to visualize the outflow tracts and the subaortic part of the interventricular septum.

The left outflow tract (also called left long axis) is obtained from the 'four-chambers' view, by rotating the transducer toward the right fetal shoulder.

In the left long axis, the following structures can be evaluated (Fig. 17):

- The connection between the left ventricle and the aorta

- The integrity of the subaortic part of the interventricular septum

- The presence and function of the aortic valve.

By rotating the transducer from the long axis view toward the fetal head, the right short axis view can be obtained showing the right outflow tract.

In the right short axis view, the following structures can be seen (Fig. 18):

- The connection between the right ventricle and the pulmonary artery 


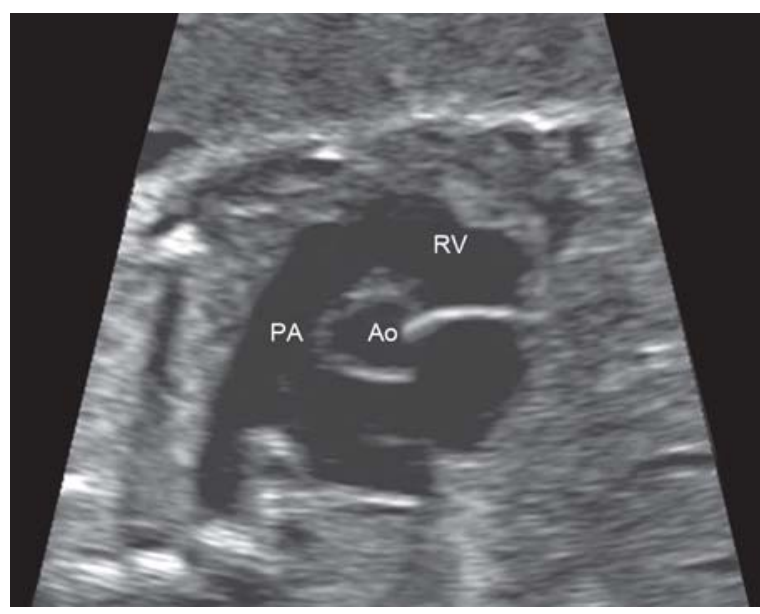

Fig. 18: Right short axis of the heart showing the pulmonary artery (PA) emerging from the right ventricle (RV) and crossing the aorta (Ao)

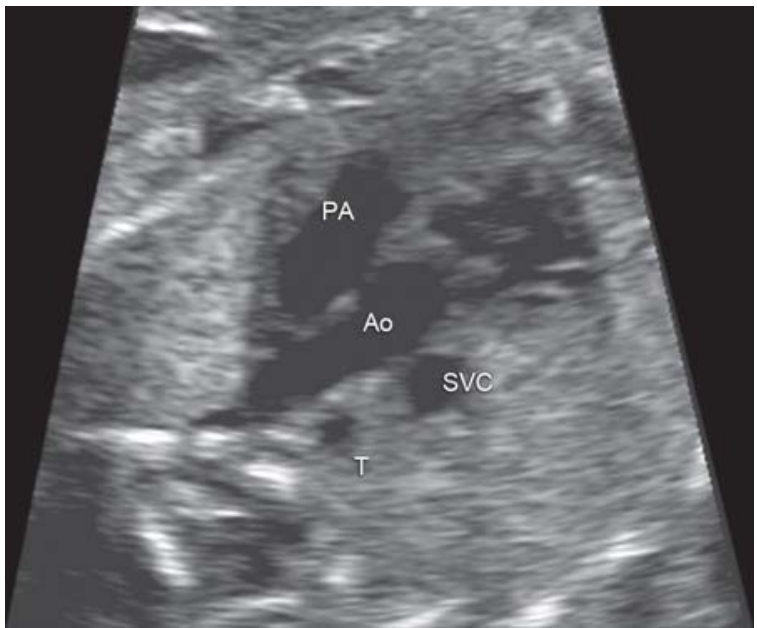

Fig. 19: Three vessels-trachea view, showing the superior vena cava (SVC), the aortic arch (Ao), the pulmonary artery (PA) continuing in the ductal arch and the trachea $(\mathrm{T})$

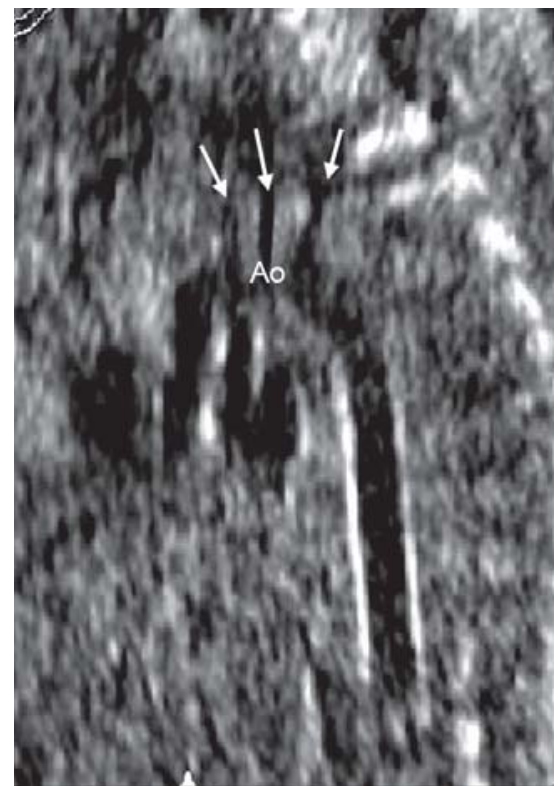

Fig. 20: Longitudinal scan of the aortic arch (Ao). The arrows point to the brachiocephalic vessels

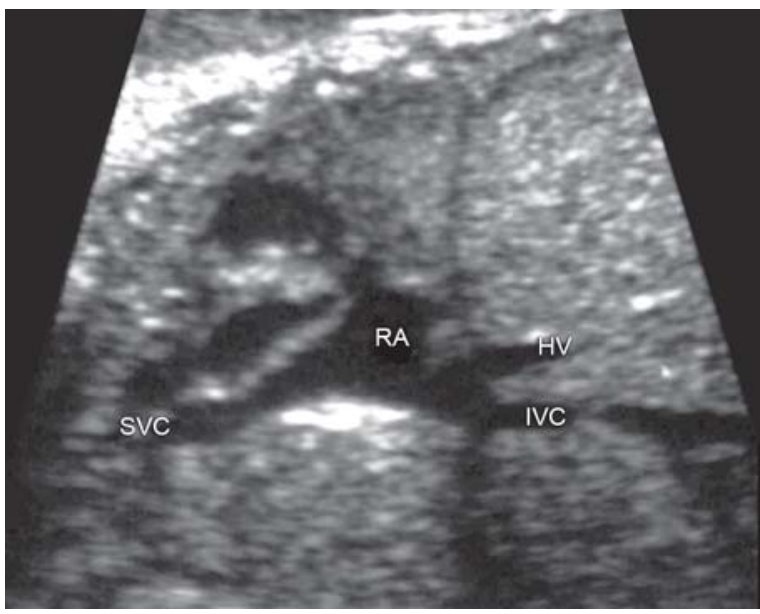

Fig. 21: Longitudinal scan showing the superior (SVC) and inferior vena cava (IVC) opening in the right atrium (RA). HV: hepatic vein

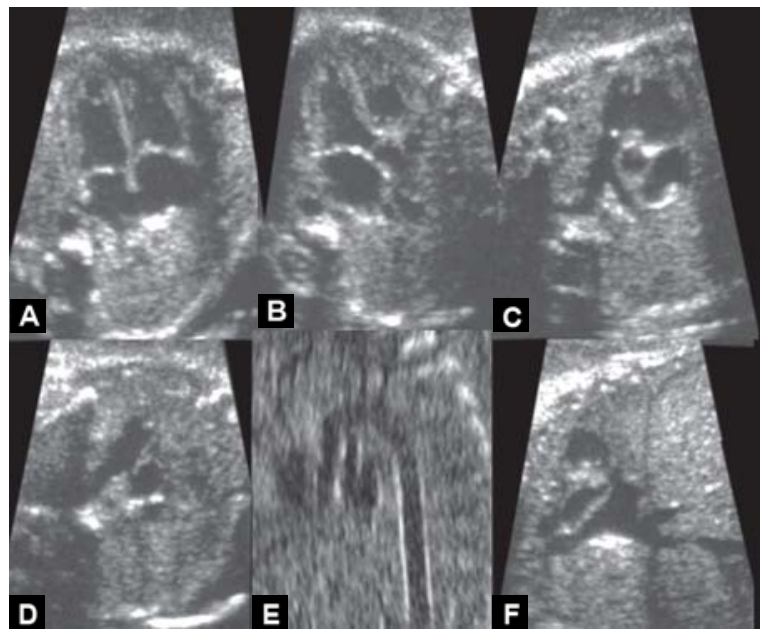

Figs 22A to F: Complete bidimensional echocardiography: (A) Fourchamber view; (B) Left ventricle outflow tract; (C) Right ventricle outflow tract; (D) Three vessels-trachea view; (E) Aortic arch; (F) Superior and inferior vena cava

- The crossover of the pulmonary artery

- The presence and function of the pulmonary valve.

An alternative way to evaluate the heart and large vessels is to obtain a correct four-chambers apical view and then to move the transducer upward to the head: the emerging aorta and the crossover of the pulmonary artery can be seen during such a movement. At the end of this excursion, the so-called 'three vessels-trachea view' (3VT view) can be obtained (Fig. 19) showing from the right to the left the superior vena cava, the aortic arch and the pulmonary artery progressing in the ductal arch; the trachea appears as a small anechoic area on the right side of the aorta and posterior to the superior vena cava.

Longitudinal scans may show the aortic arch with the brachiocephalic vessels (Fig. 20) and the superior and inferior vena cava opening in the right atrium (Fig. 21).

A complete bidimensional echocardiographic evaluation is reported in Figures 22A to F. 


\section{ABDOMEN}

The best scan to visualize the fetal abdomen is the transverse one at the level where the abdominal circumference is measured; at this level the following structures can be recognized: the intrahepatic tract of the umbilical vein on the anterior aspect, the stomach on the left side, the spine and the transverse section of the abdominal aorta on the posterior aspect (Fig. 23).

The gallbladder appears as a pear-shaped anechoic structure located in the right side of the abdomen immediately below the right lobe of the liver (Fig. 24).

The bowel during the second trimester appears as an echogenic area with irregular borders in the lower part of the abdomen (Fig. 25).

Longitudinal section of the abdomen and chest show the diaphragm as a thin hypoechoic curve line dividing the liver from the lung (Fig. 26).

Following the course of the umbilical vein, the insertion of the umbilical cord on the abdominal wall is seen (Fig. 27).

The examination of the fetal abdomen also includes the visualization of kidneys and bladder. The kidneys are visible on the high axial scan of the abdomen and appear as two echogenic structures on both sides of the spine (Fig. 28). The capsule and the pelvis show an echogenicity higher than the renal tissue; a mild physiological dilatation of the renal pelvis can sometimes be seen. In the coronal scan, the kidneys show their typical "bean” shape appearance (Fig. 29).

The visualization of the bladder is easy: It appears as a cystic median structure in the lower abdomen (Fig. 30).

Above the bladder and between the legs the penis in the male fetus (Fig. 31) and the labia majora in the female (Fig. 32) may be seen.

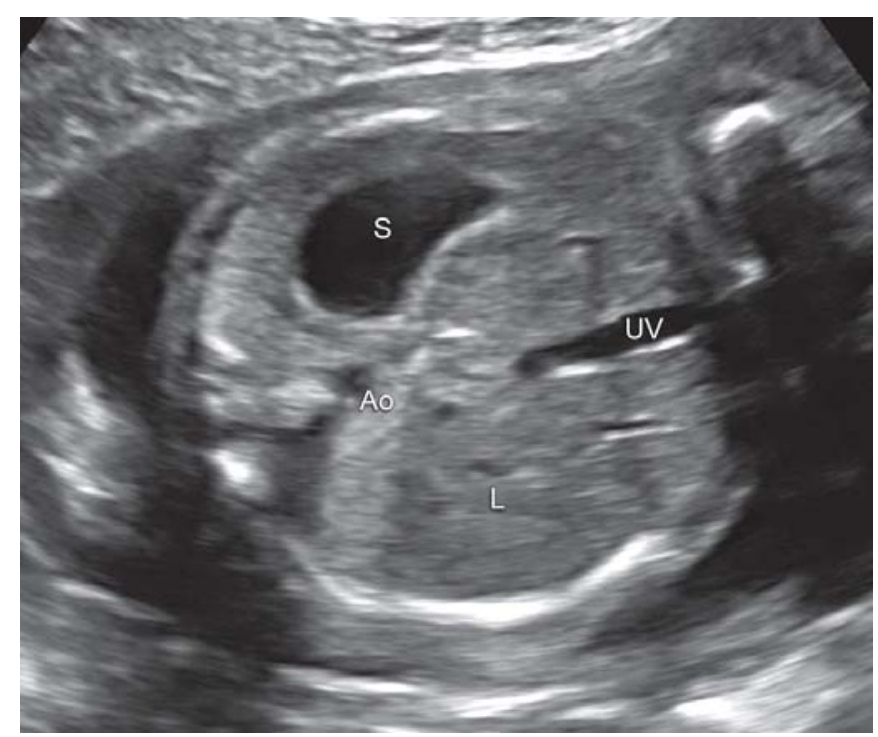

Fig. 23: Transverse scan of the abdomen for measurement of the abdominal circumference: the intrahepatic tract of the umbilical vein (UV) crossing the liver (L), the stomach (S) and the transverse section of the abdominal aorta (Ao) can be recognized

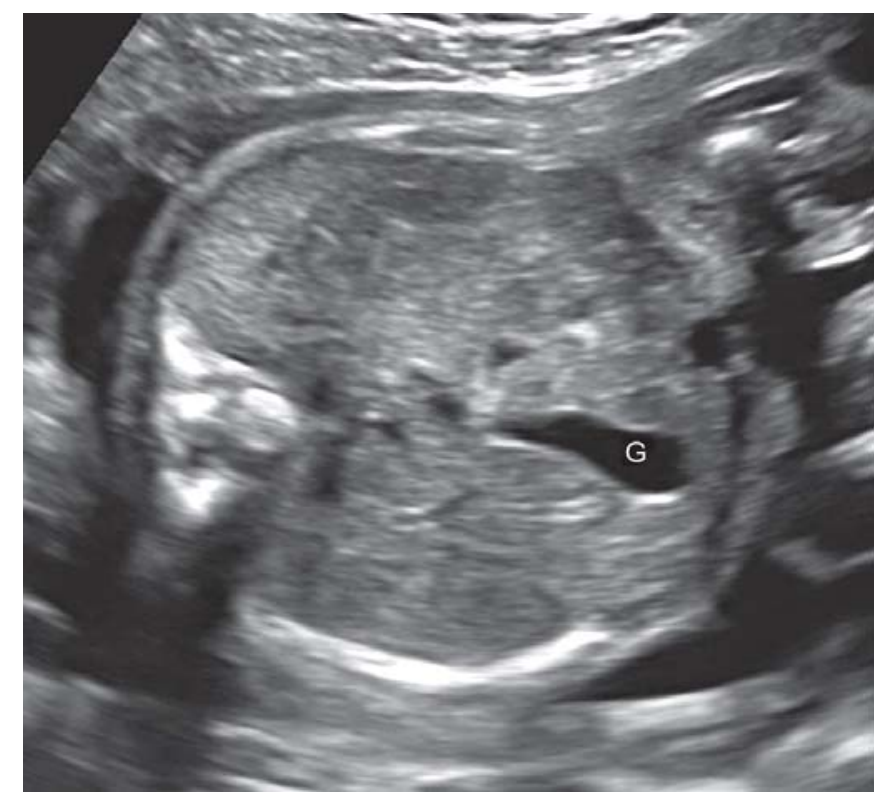

Fig. 24: The gallbladder $(\mathrm{G})$ appears as a pear-shaped anechoic structure in the right side of the liver

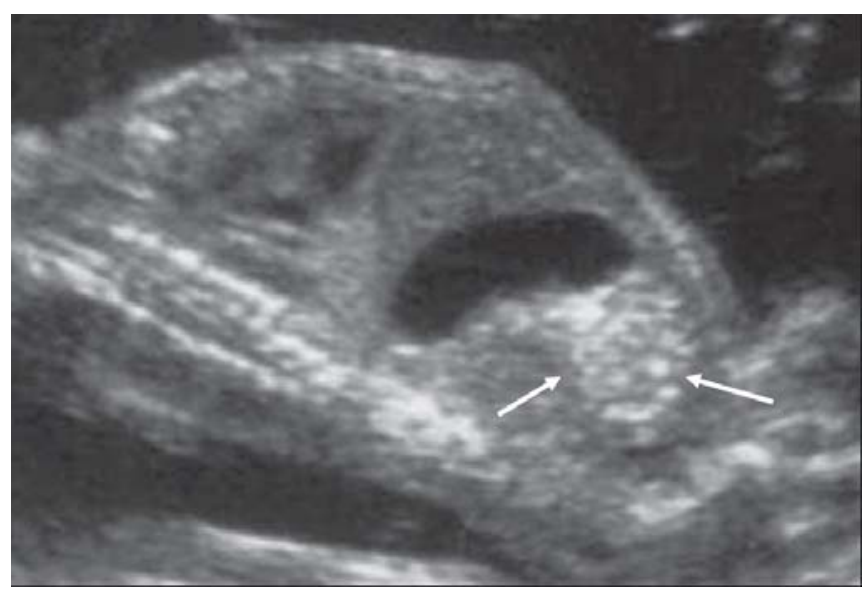

Fig. 25: The bowel appears as an echogenic area in the lower part of the abdomen (arrows)

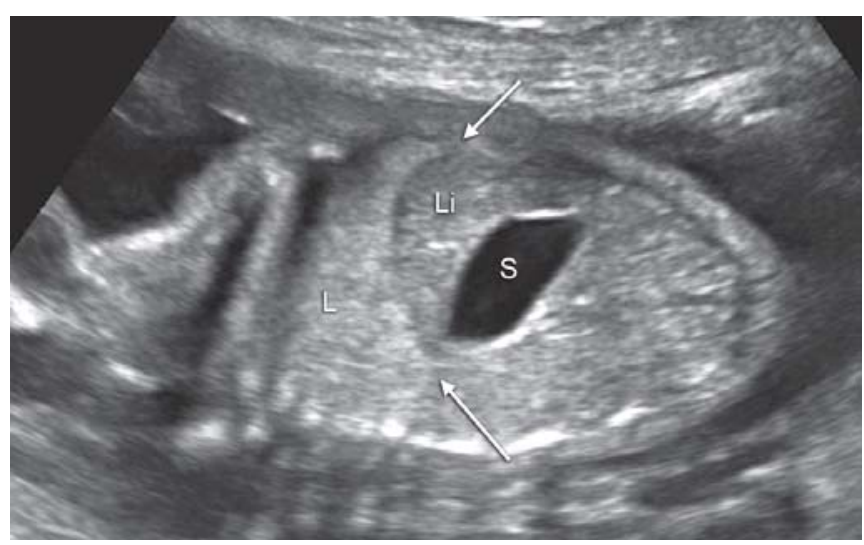

Fig. 26: The diaphragm appears as a thin curve hypoechoic line dividing the liver from the lung

\section{LIMBS}

The visualization of the limbs requires a correct understanding of the fetal lie in uterus and some skill in following their movements. The amount of amniotic fluid 


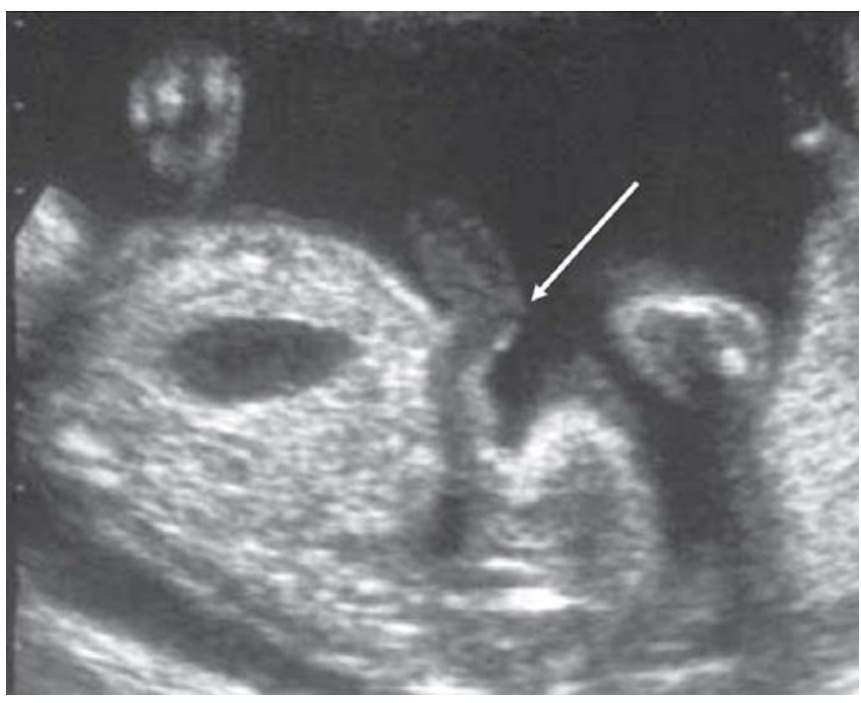

Fig. 27: Abdominal insertion of the umbilical cord

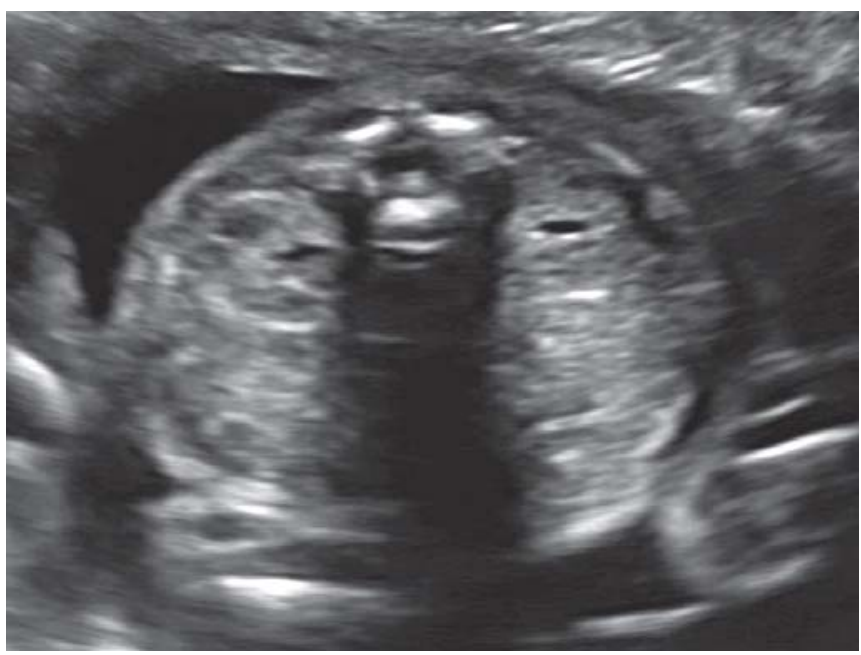

Fig. 28: Transverse section of the kidneys: They appear as two roundish moderately echogenic structure. A slight dilatation of the pelvis is frequently seen

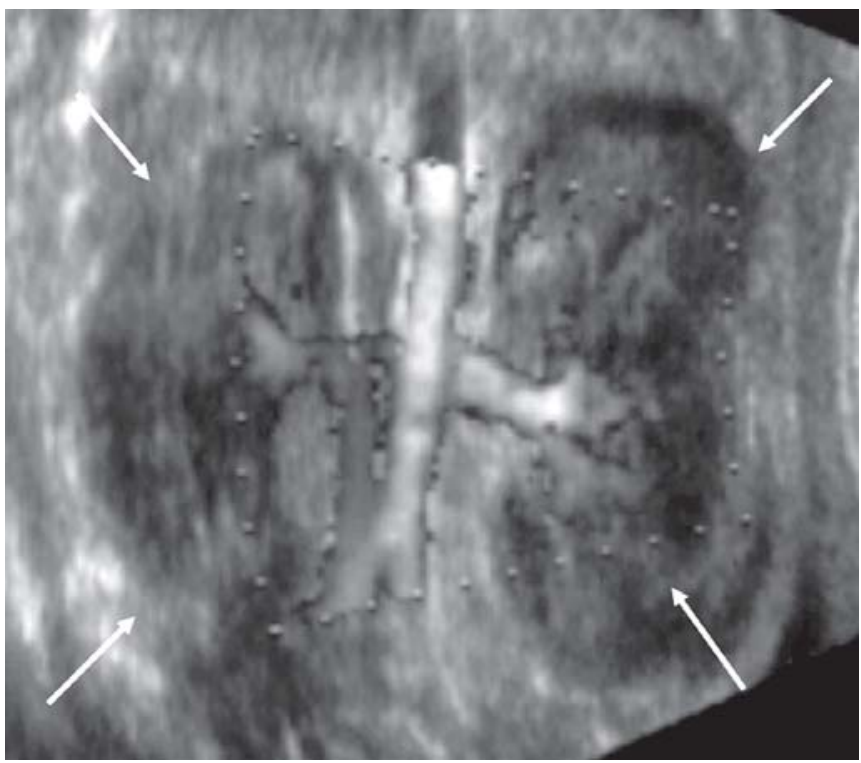

Fig. 29: Coronal section of the kidneys showing their typical bean-shaped appearance

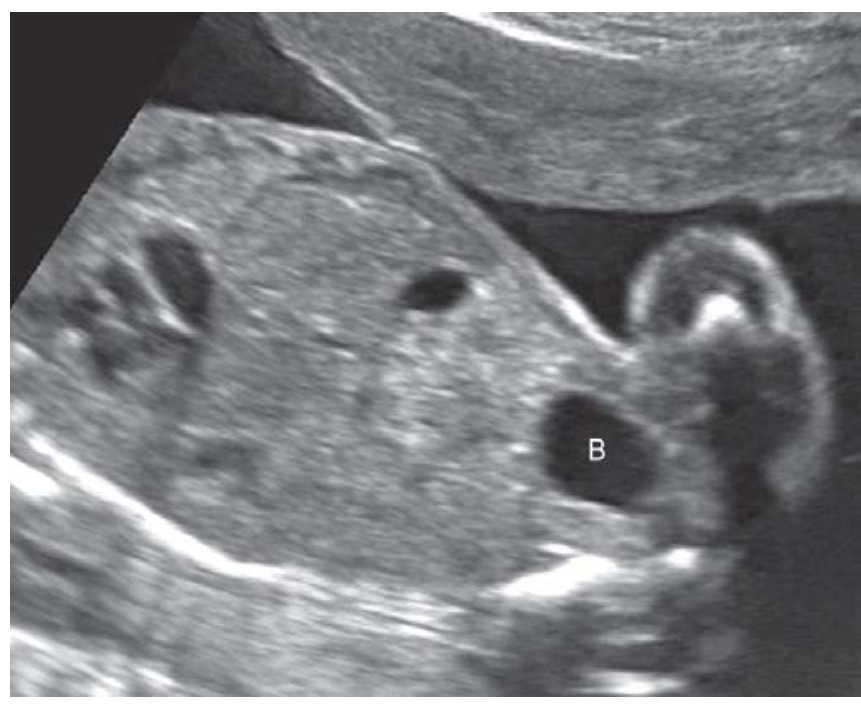

Fig. 30: The bladder (B) is easily recognized as a roundish cystic structure in the fetal pelvis

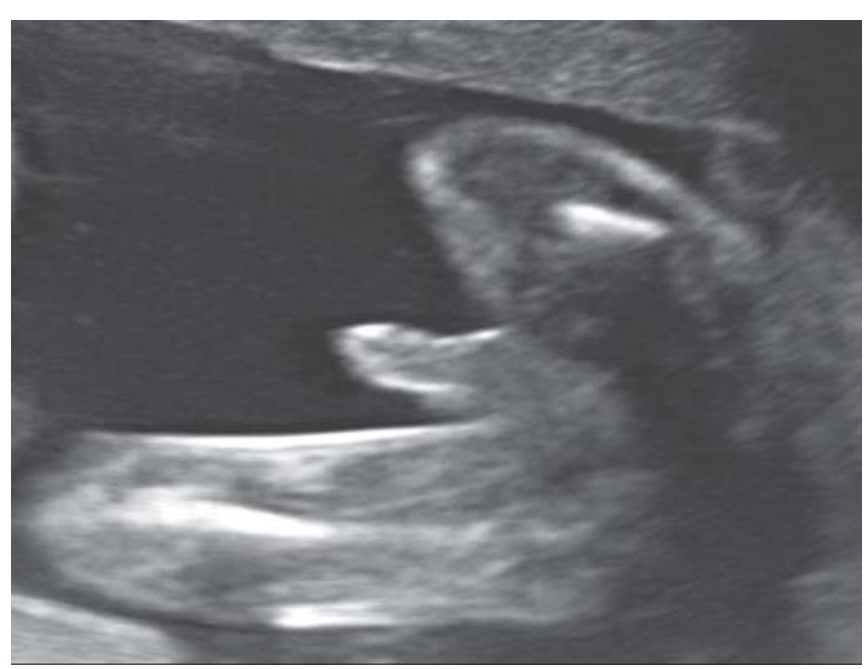

Fig. 31: Male genitalia

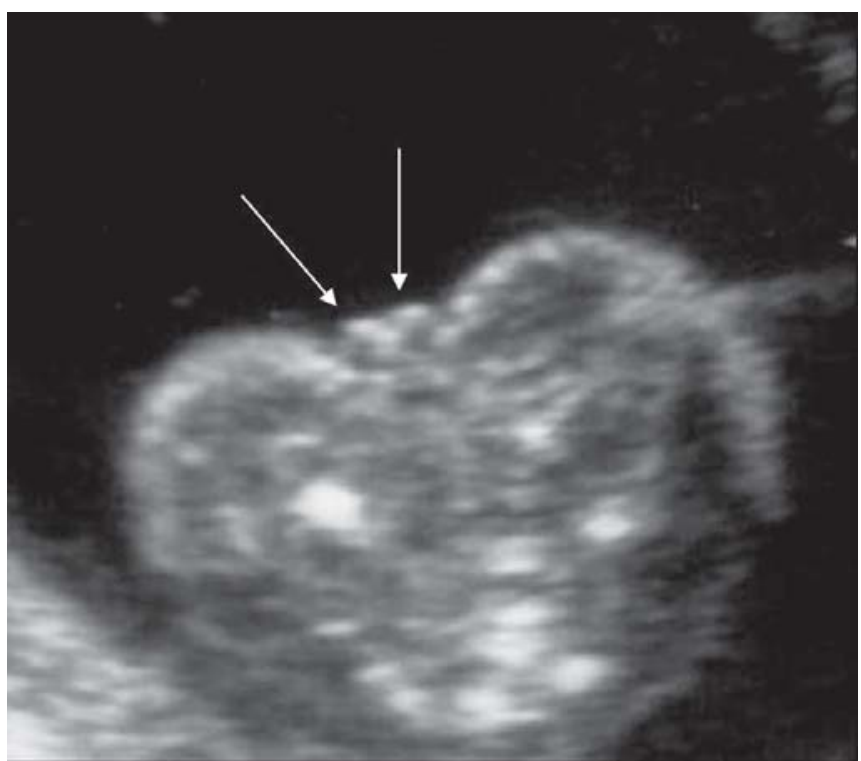

Fig. 32: Female genitalia. The arrows point to the labia majora 
is very important: In case of oligohydramnios, the recognition of the limbs becomes difficult. The long bones of the four arms may be recognized (Figs 33 and 34).

The hands and feet should also be visualized but only to define whether they are present or absent. The count of the fingers on the open hand is not always possible since the fetus usually has the hands in the closed position.

The fetal anatomical features described so far are only a part of the innumerable fetal structures that can be visualized and that will be reported in detail in another section of this book regarding the malformations of the different anatomical systems.

In order to standardize the fetal morphological examination, several scientific societies have suggested guidelines reporting the minimal standards of the fetal structures, which should be visualized during a routine scan performed during the second trimester. ${ }^{3-5}$

The guidelines of the Italian Society of Ultrasound in Obstetrics and Gynecology (SIEOG) suggest the visualization and measurement of the following structures ${ }^{3}$ (Figs 35A to $\mathrm{M}$ ):

\section{Head and Face}

- Measurement of the BPD and head circumference (it means that a correct scan of the skull and brain has been obtained showing the integrity of the calvarium, the cavum septi pellucidi, the thalami, the falx, the symmetry of the two cerebral hemispheres)

- Measurement of the atrial width (it means that the lateral ventricles have been evaluated)

- Measurement of the transverse cerebellar diameter (it means that the normality of the posterior fossa has been checked)

- Visualization of the orbits

- Visualization of the upper lip.

\section{Spine}

- Longitudinal view of the spine.

\section{Chest}

- Visualization of the lungs on a transverse scan

- Situs cardiacum

- Four-chambers view

- Left outflow tract

- Right outflow tract.

\section{Abdomen}

- Measurement of the abdominal circumference

- Visualization of the stomach and the anterior abdominal profile

- Visualization of the kidneys and bladder.

\section{Limbs}

- Visualization of the long bones of the four limbs

- Visualization of the hands and feet (present/absent) without counting the fingers

- Measurement of the femur length.

The guidelines of the American Institute of Ultrasound in Medicine (AIUM) ${ }^{4}$ suggest the visualization of the following structures:

\section{Head, Face and Neck}

- Cerebellum

- Choroid plexus

- Cisterna magna

- Lateral cerebral ventricles

- Midline falx

- Cavum septi pellucidi

- Upper lip.

\section{Comment}

A measurement of the nuchal fold may be helpful during a specific age interval to suggest an increased risk of aneuploidy.

\section{Chest}

- The basic cardiac examination includes a four-chamber view of the fetal heart

- If technically feasible, views of the outflow tracts should be attempted as part of the cardiac screening examination.

\section{Abdomen}

- Stomach (presence, size and situs)

- Kidneys

- Bladder

- Umbilical cord insertion site into the fetal abdomen

- Umbilical cord vessel number.

\section{Spine}

Cervical, thoracic, lumbar and sacral spine.

\section{Extremities}

Legs and arms: Presence or absence.

\section{Sex}

Medically indicated in low-risk pregnancies only for the evaluation of multiple gestations.

Minimum requirements for the basic fetal anatomic survey during the midtrimester of pregnancy according to 


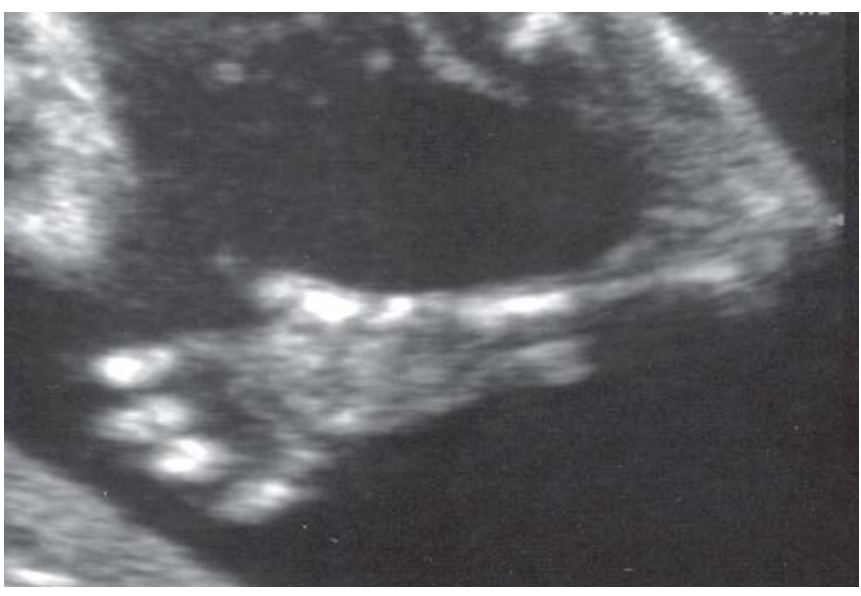

Fig. 33: Upper limb

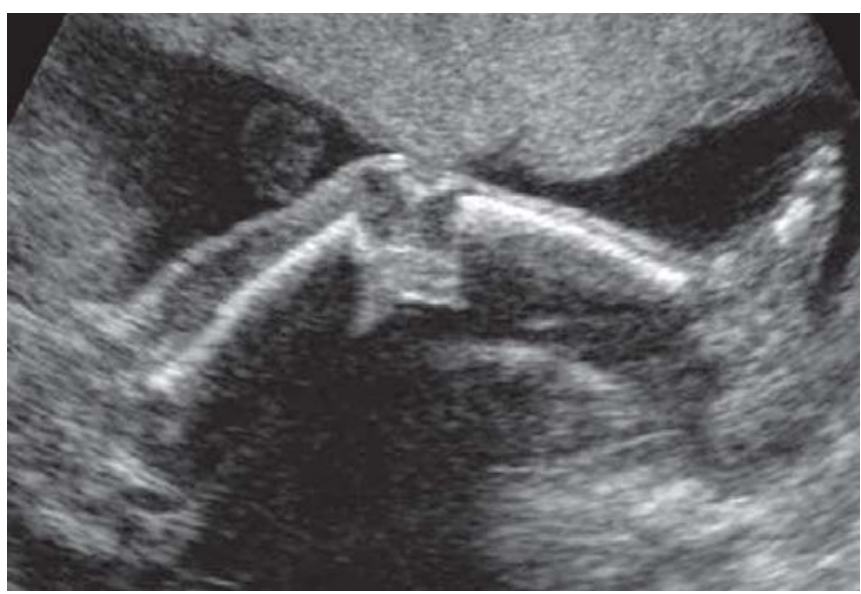

Fig. 34: Lower limb
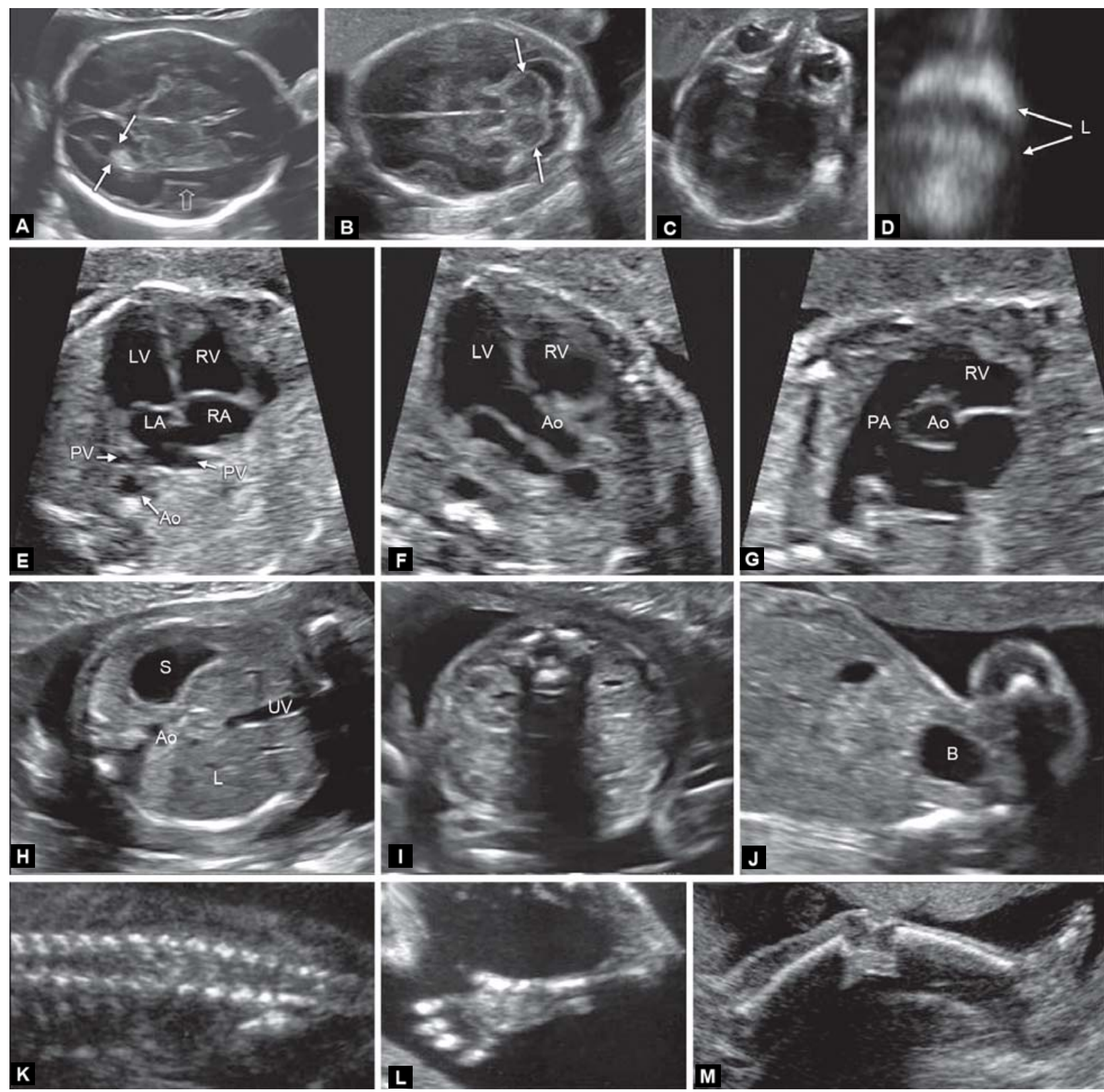

Figs 35A to M: Minimal standards for fetal anatomical survey during the second trimester required by the Guidelines of the Italian Society of Ultrasound in Obstetrics and Gynecology. (A) Transventricular view; (B) Transcerebellar view; (C) Transorbital view; (D) Upper lip; (E) Four-chambers view; (F) Left outflow tract; (G) Right outflow tract; (H) Transabdominal view with the stomach; (I) Transverse view of the kidneys; (J) Bladder; (K) Longitudinal view of the spine; (L) Upper limb; (M) Lower limb 
the guidelines of the International Society of Ultrasound in Obstetrics and Gynecology (ISUOG) ${ }^{5}$ are the following:

\section{Head}

\section{Skull}

Size, shape, integrity and bone density of the skull are visualized at the time of the head measurements and when the brain is evaluated for anatomic integrity.

\section{Brain}

Two axial planes permit visualization of the cerebral structures relevant to the anatomic integrity of the brain. These planes are commonly referred to as the transventricular and transthalamic planes. A third axial transcerebellar plane can be added to evaluate the posterior fossa. The following brain structures should be evaluated:

- Lateral ventricles (including choroid plexi)

- Cavum septi pellucidi

- Thalami

- Cerebellum

- Cisterna magna.

\section{Face}

Suggested views for minimum evaluation of the fetal face include the presence of both orbits, evaluation of the nose/ nostrils and presence of the mouth. If technically feasible, a median facial profile view can be obtained.

\section{Neck}

Detailed neck anatomy is not part of the screening examination, although this region can be evaluated in transverse and sagittal planes. This structure should be cylindrical with no protuberances, masses or fluid collections.

\section{Thorax}

The shape should be regular with a smooth transition to the abdomen. The ribs should have normal curvature without deformities. Both lungs should appear homogeneous and without evidence for mediastinal shift or the presence of masses. The diaphragmatic interface can often be visualized as a hypoechoic dividing line between the thoracic and abdominal content (e.g. liver and stomach).

\section{Heart}

Basic cardiac examination: The basic cardiac screening examination is interpreted from a four-chamber view of the fetal heart. A normal regular rate ranges from 120 to 160 beats per minute. The heart should be located in the left chest (same side as the fetal stomach) if the situs is normal. A normal heart is usually no larger than one-third the area of the chest and without pericardial effusion. The heart is normally deviated about $45 \pm 20^{\circ}$ (2 SD) towards the left side of the fetus.

Extended basic cardiac examination: This includes the aortic and pulmonary outflow tracts and can increase the detection rate for major cardiac malformations above those achievable by the four-chamber view alone. These additional views to the basic examination are more likely to identify conotruncal anomalies. Normal great vessels are approximately equal in size and should cross each other as they exit from their respective ventricular chambers.

Some investigators have described an optional 'threevessels and trachea view' that may also be useful for evaluating the pulmonary artery, ascending aorta and right superior vena cava in terms of their relative sizes and anatomic relationships.

\section{Abdomen}

The situs of abdominal organs should be determined. The fetal stomach should be identified in its normal position on the left side.

The following should be routinely imaged:

- Stomach

- Bowel

- Umbilical cord insertion and intact abdominal wall

- Three-vessel cord.

\section{Kidneys and Bladder}

The fetal bladder and both kidneys should be identified. If either the bladder or renal pelvis appear enlarged, a measurement should be documented. Persistent failure to visualize the bladder should prompt a referral for a more detailed assessment.

\section{Spine}

The detailed examination of the fetal spine requires expertise and meticulous scanning, and the results are heavily dependent upon the fetal position. Therefore, a full detailed evaluation of the fetal spine from every projection is not a part of the basic examination. The most frequent of the severe spinal abnormalities, open spina bifida, is usually associated with abnormal intracranial anatomy such as a no visualized cisterna magna. However, a longitudinal section of the fetal spine should always be obtained because it may reveal at least in some cases, other spinal malformations including vertebral abnormalities and sacral agenesis. 


\section{Limbs and Extremities}

The presence or absence of both arms/hands and both legs/ feet should be documented using a systematic approach. Counting fingers or toes is not required as part of the basic scan.

\section{Genitalia}

Characterization of external genitalia, to determine fetal gender, is not considered mandatory in the context of a midtrimester routine scan. Reporting of gender should be considered only with parental consent and in the context of local practices.

Although many countries have already developed local guidelines for the practice of fetal ultrasonography, there are still many areas of the world where they have not been implemented. This can be related to the availability of qualified examiners and equipment, local practices, legal considerations and costs related to reimbursement. Nonetheless, a WHO Study Group ${ }^{6}$ stated: 'Worldwide, it is likely that much of the ultrasonography currently performed is carried out by individuals with in fact little or no formal training.' It is desirable, therefore, that the use of guidelines in performing midtrimester anomaly scan becomes a common worldwide practice. It in fact allows the performance of a good level basic examination with the correct selection of patients which should be referred to a second level center.

\section{REFERENCES}

1. Kalousek DK, Lau AE. Development of the embryo, fetus and placenta. In: Dimmick JE, Kalousek DK (Eds). Developmental pathology of the embryo and fetus. New York: JB Lippincott Co 1992;1-25.

2. Staudach A. Fetal echo-anatomy. Berlin:Springer-Verlag 1989.

3. SIEOG. Linee Guida 2010. www.sieog.it.

4. AIUM practice guidelines for the performance of obstetric ultrasound examination. www.aium.org, 2007.

5. ISUOG practice guidelines for performance of the routine midtrimester fetal ultrasound examination. www.isuog.org 2010.

6. World Health Organization. Training in diagnostic ultrasound: Essentials, practice, and standards. Geneva, World Health Organization (WHO Technical Report Series, No. 875);1998.

\section{ABOUT THE AUTHORS}

\section{Vincenzo D'Addario (Corresponding Author)}

Department of Obstetrics and Gynecology, University of Bari, Bari Italy, e-mail: v.daddario@gynecology4.uniba.it

\section{Vincenzo Pinto}

Department of Obstetrics and Gynecology, University of Bari Bari, Italy

\section{Luca Di Cagno}

Department of Obstetrics and Gynecology, University of Bari Bari, Italy

\section{Armando Pintucci}

Department of Obstetrics and Gynecology, University of Bari Bari, Italy

\section{Angela Cristina Rossi}

Department of Obstetrics and Gynecology, University of Bari Bari, Italy 